\title{
Early postnatal development of the lumbar vertebrae in male Wistar rats: double staining and digital radiological studies
}

\author{
F. Burdan 1, 2, M. Błaszczak-Szalak¹,*, I. Różyło-Kalinowska³, R. Klepacz4, W. Dworzański , \\ T.K. Różyło ${ }^{5}$, J. Dudka ${ }^{4, \# \text {, J. Szumiło }}{ }^{4}$ \\ ${ }^{1}$ Human Anatomy Department, Medical University of Lublin, Poland \\ ${ }^{2}$ Radiology Department, St. John's Cancer Centre, Lublin, Poland \\ 3 Independent Unit of Propaedeutic of Dental and Maxillofacial Radiology, Medical University of Lublin, Poland \\ ${ }^{4}$ Clinical Pathomorphology Department, Medical University of Lublin, Poland \\ ${ }^{5}$ Dental and Maxillofacial Radiology Department, Medical University of Lublin, Poland \\ *Currently Miedzylesie Hospital, Miedzylesie-Warsaw, Poland \\ \#Currently Medical Biology Unit, Medical University of Lublin, Poland
}

[Received: 22 February 2015; Accepted: 30 March 2015]

\begin{abstract}
The aim of the study was to evaluate the physiological developmental changes of male rats' lumbar vertebrae during the first 22 days after birth. Morphology and mineralisation of lumbar vertebrae were evaluated using double-staining and digital radiography system, which allowed vertebral width and optical density to be determined. Pup weight, crown-rump length, body mass index and vertebral width increased during postnatal period and significantly correlated with their age. Bone mineralisation, as measured by optical density, did not show any significant differences. The complete fusion of the primary ossification centres had a cranio-caudal direction and started on day 19 after parturition but was incomplete by day 22. It could be concluded that, unlike significant age-related increase of vertebral size, mineralisation was only slightly elevated during evaluated postnatal period. The method described is supplementary to alizarin red S staining as it provides both qualitative and quantitative data on mineralisation in a similar manner to micro computed tomography but does not allow 3 dimensional and microarchitecture examination. (Folia Morphol 2016; 75, 1: 1-13)
\end{abstract}

Key words: bone mineralization, ossification, vertebral column, postnatal development

\section{INTRODUCTION}

Bone evaluation is an obligatory step in regulatory reproductive toxicity studies, particularly in $C$ and D stages according to the classification of International Conference on Harmonisation of Technical Requirements for Registration of Pharmaceuticals for Human Use $(\mathrm{ICH})[2,19]$ as well as other guidelines like Environmental Protection Agency (EPA) [15] and Organisation for Economic Co-operation and Devel- opment (OECD) [26]. The skeleton can be examined by using various staining methods. Methods which use alizarin red $\mathrm{S}$ staining are simple, fast, well-known and widely applied and allow bone morphology to be studied. However, cartilaginous elements cannot be examined in details [1, 3, 6-11, 21, 23, 32, 33]. Unlike single staining, double staining with alizarin red $\mathrm{S}$ and alcian blue permits evaluation of both bone and cartilaginous elements but since it requires removal

Address for correspondence: Prof. F. Burdan, MD PhD, ERT, Experimental Teratology Unit, Human Anatomy Department, Medical University of Lublin, ul. Jaczewskiego 4, 20-090 Lublin, Poland, tel: +48 6037676 49, e-mail: fb3@wp.pl 
of the skin, it is regarded as a labour-intensive and time-consuming technique.

Currently, foetal bone morphology, including shape and mineralisation, may be evaluated by various radiological methods, as well. The most commonly applied one is micro computed tomography $(\mathrm{mCT})$, and various densitometric and classical radiological methods $[5,7,14,17,18,25,30,31]$. Rarely, micro magnetic resonance (mMR) and positron emission tomography connected with CT (PET-CT) or MR (PET-MR) may be also applied [4]. From classical radiological methods, digital radiography was successfully applied in reproductive studies. It was helpful in evaluation of mineralisation of vertebral column in animals exposed in-utero and in an early postnatal period to different xenobiotics, e.g. valproic acid, non-steroidal anti-inflammatory drugs [5-7].

The aim of the current study was to evaluate the physiological age-related changes of rats' lumbar vertebrae during the first 3 weeks of postnatal life (birth till weaning) and to prove usefulness of the applied methodology in developmental studies.

\section{MATERIALS AND METHODS}

The study protocol was approved by the First Local Bioethical Committee of Medical University of Lublin (Lublin, Poland; guidelines \#133/2001).

Sexually mature albino rats of Wistar CRL:(WI) WUBR strain (12-15 weeks of age) obtained from an accredited commercial breeder (Warsaw-Rembertow, Poland) were selected for the study. Animals were acclimated for at least 2 weeks, housed and maintained in an animal care facility, as described before $[5,7]$. Filtered municipal (Lublin, Poland) tap water and standard laboratory LSM ${ }^{\circledR}$ rat diet (Agropol; Motycz, Poland) were provided ad libitum. On mating days, females of the proper weight (range 200-250 g) were placed in cages with males (5:2) for approximately $14 \mathrm{~h}$. The following morning, a vaginal smear was prepared to determine if copulation had occurred. The day when sperm was found was designated gestation day 1 and females were individually placed in plastic cages. All animals delivered spontaneously and were not exposed to any xenobiotic during pregnancy or lactation. The day of parturition was set as the first day of postnatal life.

To exclude any potential hormonal and developmental sex differences, only male pups were examined. Since physiological changes were studied, all evaluated animals aged 1-22 days without any external developmental anomalies and signs of intrauterine growth retardation (such as low body weight and crown-rump length) were randomly selected from untreated litters. Each animal was taken from litters with initial number of offspring over 8 and without any developmental abnormalities among pups. From 1 litter, only up to 3 pups were selected with at least 4 day interval period. Each postnatal day up to 10-12 animals were examined.

The pups weight and crown-rump length were checked. The body mass index (BMI) was calculated, as supplementary developmental marker [8].

After pentobarbital (BIOWET; Pulawy, Poland) euthanasia, each offspring was eviscerated and partly skinned to expose lumbar and adjacent parts of the vertebral column. The lumbar vertebrae were selected since they are relatively large and are not covered by other calcium containing structure on radiographs, like on the cervical, thoracic and sacral level, that are visible on the same level of hyoid, sternum/ribs and hip bones, respectively. Previously described digital radiological method for quantitative bone mineralisation assessment was applied to evaluate morphology and mineralisation of vertebrae [5-7]. Radiographs were taken using a self-calibrating digital radiography system (Prostyle Intra X-Ray Unit; Planmeca, Finland), operating at $70 \mathrm{kV}$ and $0.02 \mathrm{mAs}$ with a multiple-use $3 \times 4 \mathrm{~cm}$ photostimulable storage phosphor plate image receptor. Each examined animal was placed in prone position in the centre of the plate. The central X-ray beam was perpendicular to the plate. The distance between the examined plate and X-ray lamp was set at $0.25 \mathrm{~m}$. After exposure, the plate was introduced into a dedicated commercial laser scanner (Digora, Soredex, Finland). The latent image was presented on a computer screen and stored on a hard disc. Only symmetrical radiographs were analysed. In case of an unsymmetrical picture, the exposition was repeated until correct position on the X-ray was obtained. The mean density of lumbar vertebrae (L1-L6) was analysed. The measurement line was drawn, separately for each vertebrae, between the most lateral point of the ossification centres of the corresponding left and right vertebral arches (Fig. 1). All the measurements were performed by one experienced radiologist (IRC). The grey scale level, represented as a range from 0 to 255, was used for radiographs analysis. According to general principles of the system, zero corresponds to areas where no attenuation of radiation occurred and such areas 


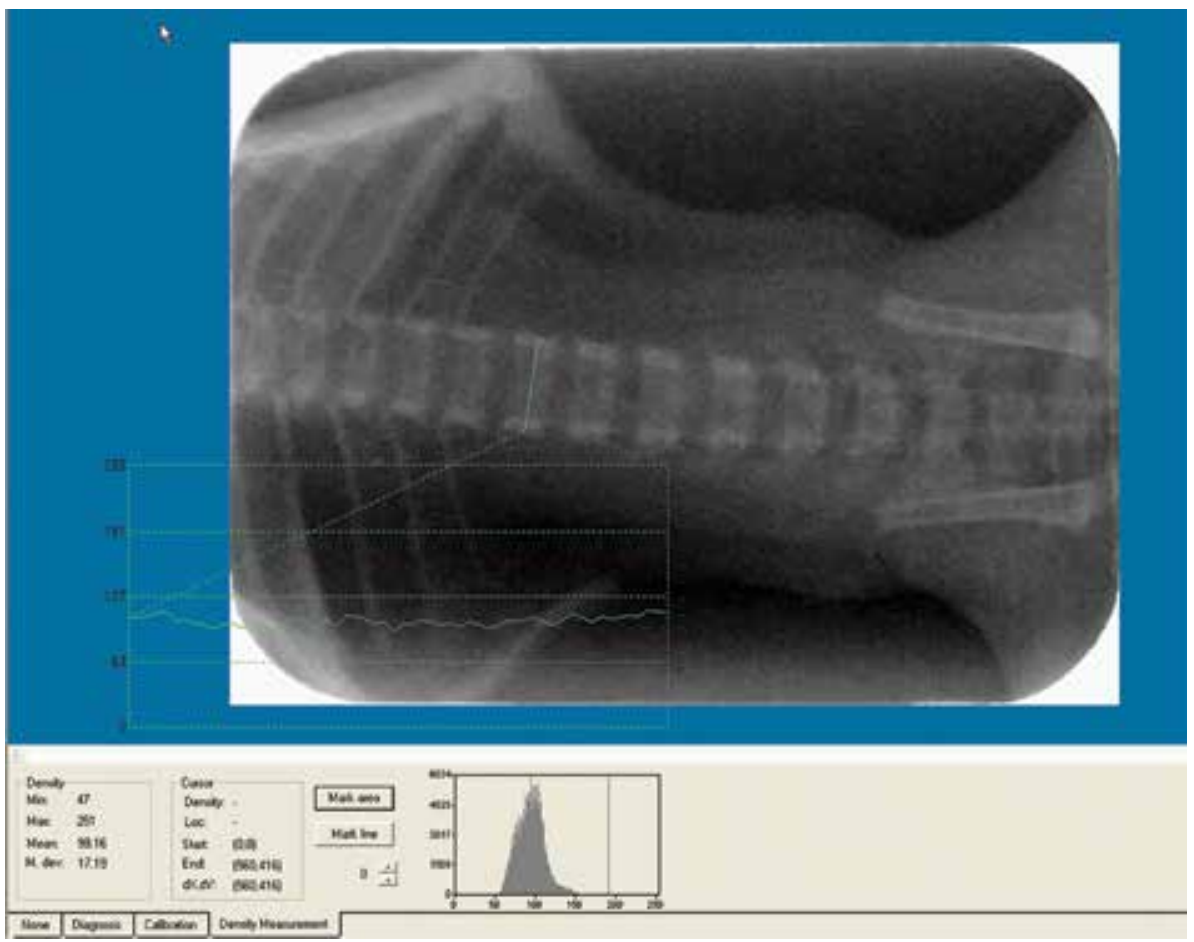

Figure 1. Density measurement window. Sample of the density examination of the first lumbar (L1) vertebra of 16-day old rat pup. The left, lower corner presents density profile along a measurement line marked at the level of the L4 vertebra. Lower left corner: min, max, and mean — minimum, maximum and mean density recorded along the line; M. dev — mean deviation; Cursor window: Density — density value corresponding to the position of the cursor; Loc - location of the cursor calculated from top left corner of the image and shown as pixels; Start, End - starting and ending points of the measurement line (calculated from top left corner of the image and shown as pixels); $d X d Y$ — length of measurement line (in pixel). The box in the centre at the bottom of figure shows the histogram representing the distribution of density along the measurement line.

are presented as black, while pixels with 255 values are presented as white and correspond to areas in which the ionising radiation has been fully absorbed on X-ray by the radiographed object.

After radiological examination, the animals were completely skinned, dehydrated and double-stained using alcian blue and alizarin red-S $[8,33]$. All reagents were at laboratory or higher grades and came from Sigma Chemical Co. (Saint Louis MO, USA), except for ethanol ( $\mathrm{POCH}$; Lublin, Poland). Specimens stored in glycerine were examined under a stereo-dissection microscope. Lumbar vertebrae were separated and the fusion of ossification centres of the vertebral body and arches was evaluated separately for each lumbar segment. Development changes of vertebral processes were also revealed but such data are not presented in this paper since they were not easily visible on radiographs.

The unit for statistical measurement was pup (litter, since single pups was taken from litter each day; see comments above). The mean density of the lumbar region was calculated individually for all the pups using density data of each vertebra (L1-L6) measured on the same $X$-ray with the following formula $[(\mathrm{L} 1+\mathrm{L} 2+\mathrm{L} 3+\mathrm{L} 4+\mathrm{L} 5+\mathrm{L} 6) / 6]$. Homogeneity of the optical vertebral density was analysed using the Kolmogorov-Smirnov test. Because of normal distribution, the data were analysed by ANOVA and followed by the Duncan test. The nominal scale measures were analysed by $\chi^{2}$ test with Yates' correction for independence of differences among the different age groups. Correlations were analysed by Spearman's rank correlation coefficient. The 0.05 level $(p<0.05)$ of probability was used as the criterion of significance.

\section{RESULTS}

One pup was withdrawn from the study group on day 5, due to occult rachischisis, limited to vertebral arches of $\mathrm{C} 3-\mathrm{C} 5$ and detected on the stained specimen. The remaining animals presented wellformed skeleton without any malformations. Lack of developmental variations was found in the vertebral column. The age-related narrowing of blue-staining cartilaginous elements with concomitant increase of 


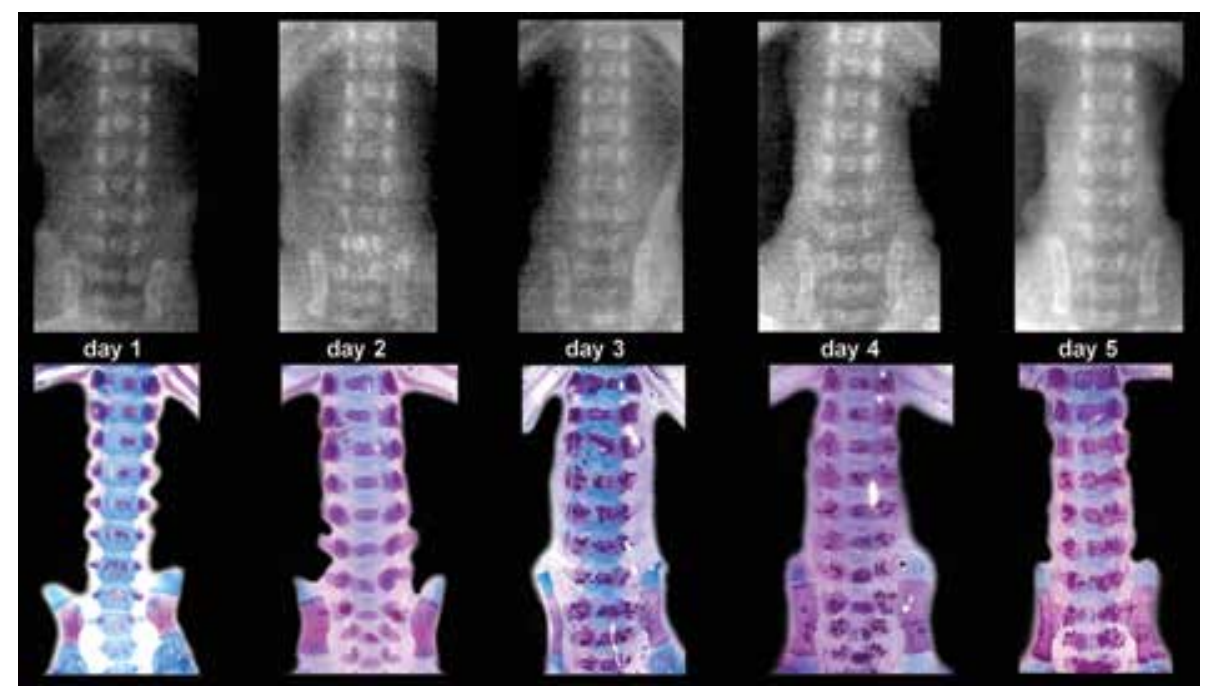

Figure 2. X-ray and the corresponding double-staining specimen of the lumbar part of vertebral column of rat male pups aged 1-5 day.

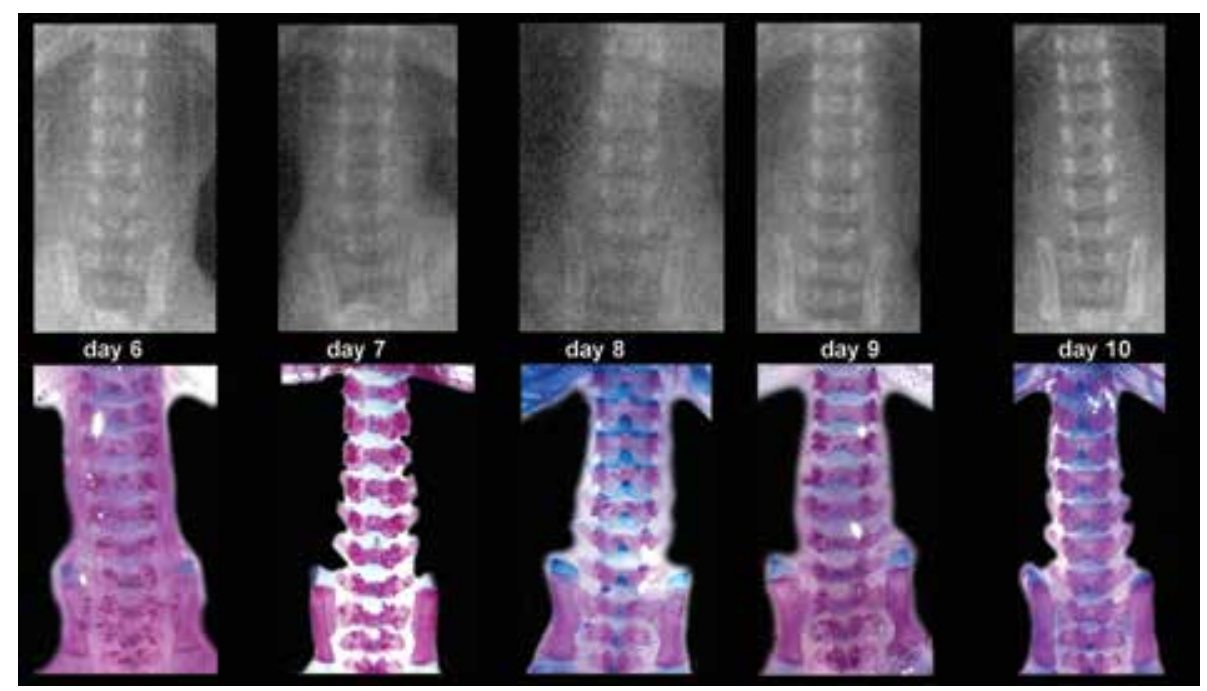

Figure 3. X-ray and the corresponding double-staining specimen of the lumbar part of vertebral column of rat male pups aged 6-10 day.

red-staining bony structures was seen in the examined population. On the corresponding $X$-ray, an increase of vertebral shadowing with concomitant increase of its width was observed (Figs. 2-6).

After separation of the lumbar part of the vertebral column, a total fusion of primary ossification centres was seen for the first time in selected animals on day 19 in L1-L3 vertebrae. The incidence increased with age, but on the last day of observation (day 22) incomplete fusion of all vertebrae was observed in a proportion of pups (Fig. 7).

Pup weight, length and BMI increased during postnatal period, and significantly correlated with their age (Fig. 8, Table 1). However, significant in- crease of pups' weight in comparison with the previous day was observed only on day $11,14,15,17$, 18,20 and 22 . In case of length such differences were found on day $3,5,11,14,15,17,18$ and 22 . One day differences were also seen for BMI on the postnatal day 7, 11, 20 and 22. A 2-day or longer interval differences were always significant.

The width of lumbar vertebrae significantly correlated with pups' age (Fig. 9). However, differences between each vertebrae in the proper day were insignificant but decreased caudally (Table 2). Based on the Spearman rank value, it could be stressed that the highest developmental differences of vertebral width were observed for upper ones: $L 1>L 2>L 3>L 4>L 5>L 6$ 


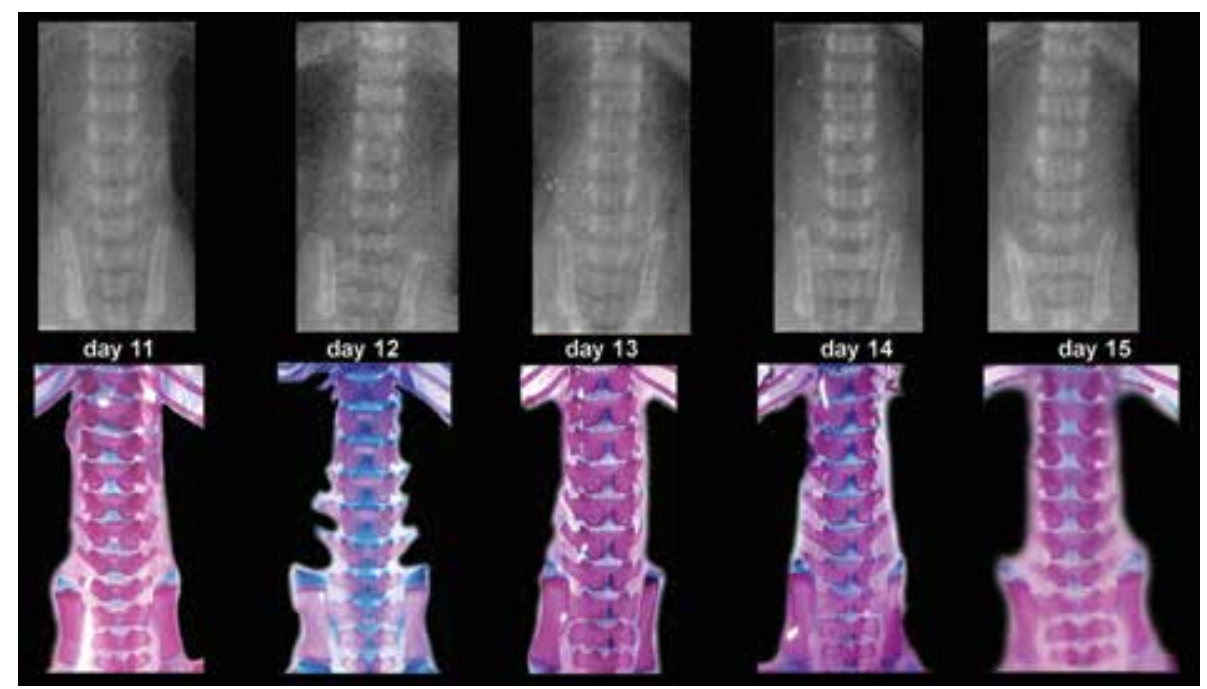

Figure 4. X-ray and the corresponding double-staining specimen of the lumbar part of vertebral column of rat male pups aged 11-15 day.

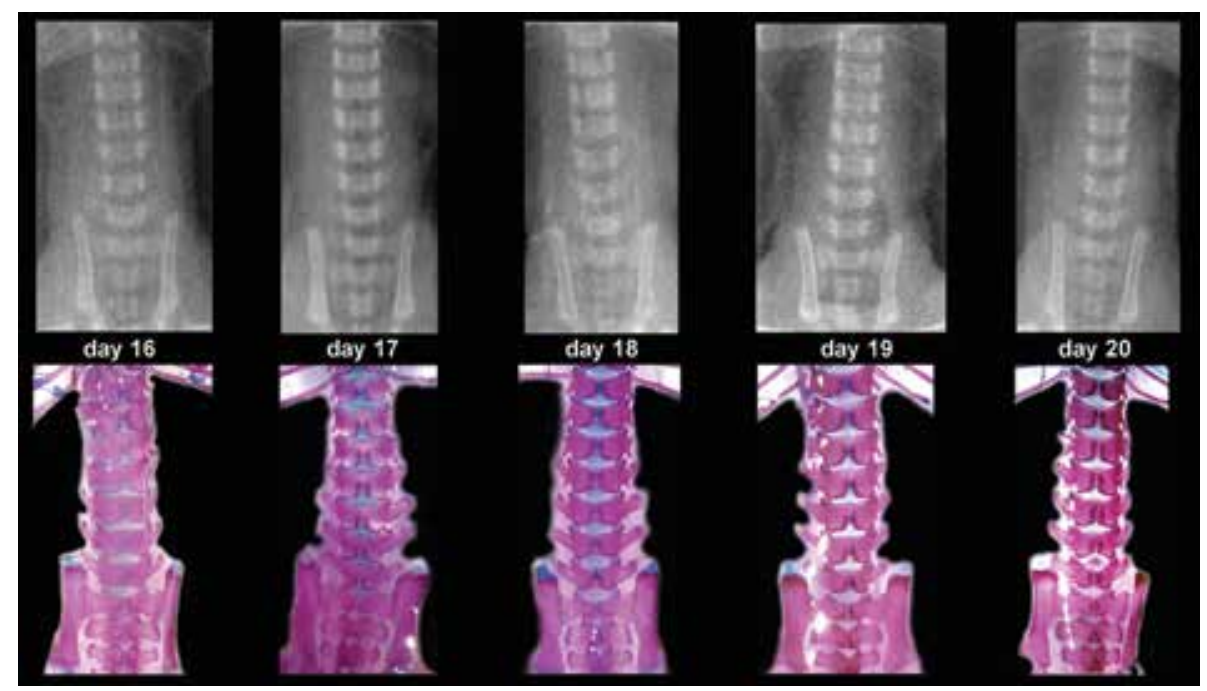

Figure 5. X-ray and the corresponding double-staining specimen of the lumbar part of vertebral column of rat male pups aged 16-20 day.

(Fig. 9). A significant one-day differences of vertebral width were usually observed on day 2, 5, 7 and 11 for L1-L4, and also on day 22 for L1, on day 14 and 22 for L2, on day 14,18 and 22 for $L 3$, as well as on day 18 for L4 (Table 2). In case of L5 and L6 they were revealed only on day 7 and 11 as well as 2 and 10, respectively. Similar to pups' weight and length, two-day or longer interval differences were always significant.

The optical density of lumbar vertebrae did not show any notable differences during first 22 days of postnatal life (Table 3). However, not significant positive correlation with an age was found (Fig. 10). The only consistent change was decrease on day 3 and increase on day 22, when compared with the previous day (Table 3). The bone density usually elevated also on days 2 and 7, and dropped on days 20 and 21 . Nevertheless, significant differences were seen only for L1 on day 2 and 7 , as well as for L2 on day 2 . In spite of the increase on day 2 and 7 , equalisation of vertebral density with the value of the first postnatal day for $\mathrm{L} 4, \mathrm{~L} 5$ and $\mathrm{L} 6$ took place on day 17 , while for L1, L2 and L3 on day 18. In older pups an insignificant density increase was observed, but with exception of days 20 and 21. Interestingly, using simultaneously analysis, until day 18 the highest mean density for the whole lumbar part of vertebral column was found 


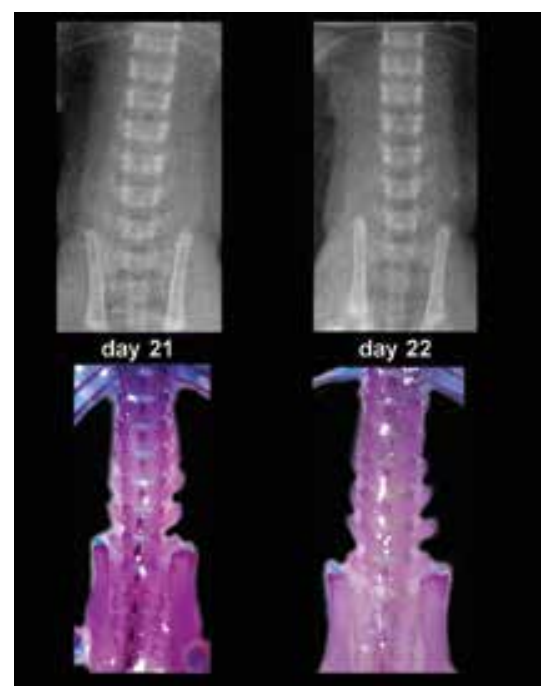

Figure 6. X-ray and the corresponding double-staining specimen of the lumbar part of vertebral column of rat male pups aged 21-22 day.

for animals with high BMI, while later the highest value was observed among individuals with a lower BMI (Fig. 11).

\section{DISCUSSION}

The applied radiological quantitative analysis showed lack of any important changes of the rat lumbar vertebrae mineralisation in the first 3 weeks of postnatal period. The only exception was significant decrease of optical density observed on the second post-parturition day. However, it may be a consequence of adaptive changes that physiologically take place after delivery in newborn, since $\mathrm{Ca}^{++}$ may be released from pup's skeleton to keep homeostasis without any direct maternal compensation [22]. On the other hand, an increase of vertebrae mineralisation (as measured by increase in optical density) was revealed on the last day of observation that was preceded by an insignificant decrease in optical density on days 20 and 21 . Such changes may initiate the final mineralisation process since most of the examined vertebrae were already well-formed. To prove such hypothesis other studies with older offspring are recommended. As expected, a logical and physiological correlation was proved between animal age and their weight, length and vertebral width.

Unlike the appendicular skeleton, development of the vertebral column in experimental animals has not been evaluated in details. However, based on the rat's alizarin red $\mathrm{S}$ staining data, mineralisation process of various skeletal parts has a different mechanism [1]. In case of 19 day old foetuses no signs of mineralisation on cervical and coccygeal vertebrae were seen. Moreover, primary ossification centres on the thoracic and sacral vertebrae were not fully formed. Mineralisation increased rapidly at the end of pregnancy since on cervical and coccygeal levels, it started on day 20 but one day later all primary ossification centres were well-visible in all cervical and 4 upper coccygeal vertebrae. One day before delivery mineralisation was similar in all examined animals. Data regarding morphology of 21-day old rat foetuses was confirmed by various authors $[3,6-9,10,21,23,32,33]$ and for this reasons its evaluation is regarded as a good and sensitive marker of any pathological factors that

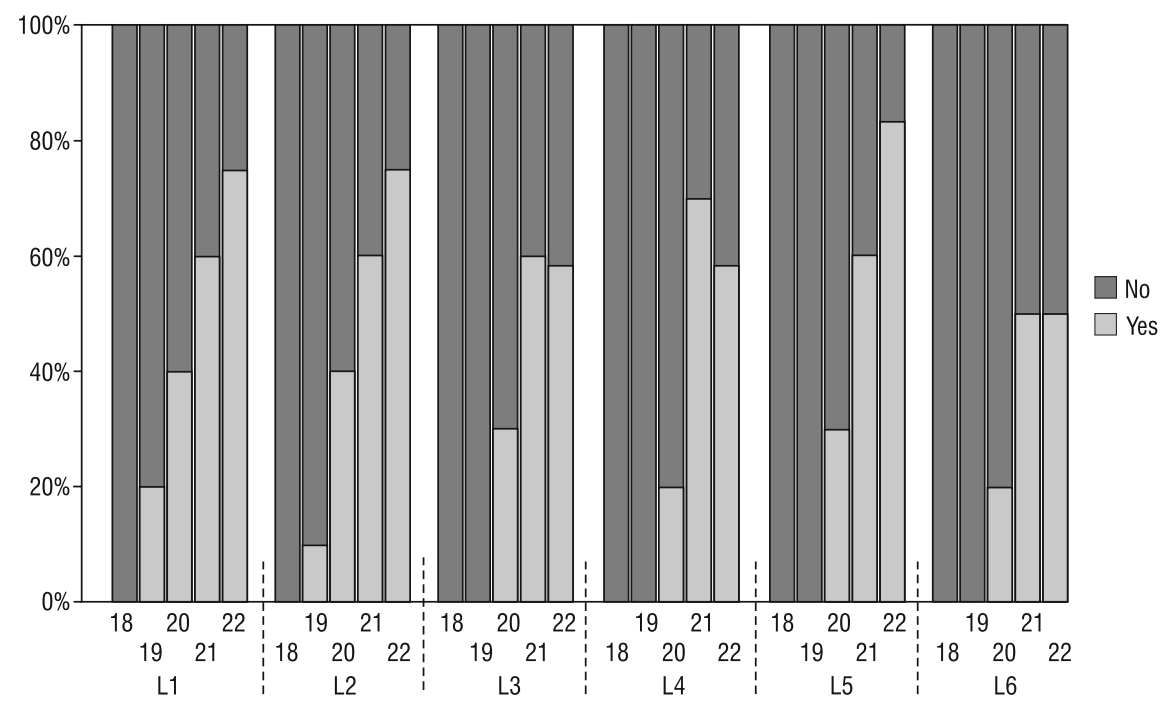

Figure 7. Incidence of total (Yes) and lack/incomplete (No) fusion of primary ossification vertebral centres in male rats aged 19-22 day. 

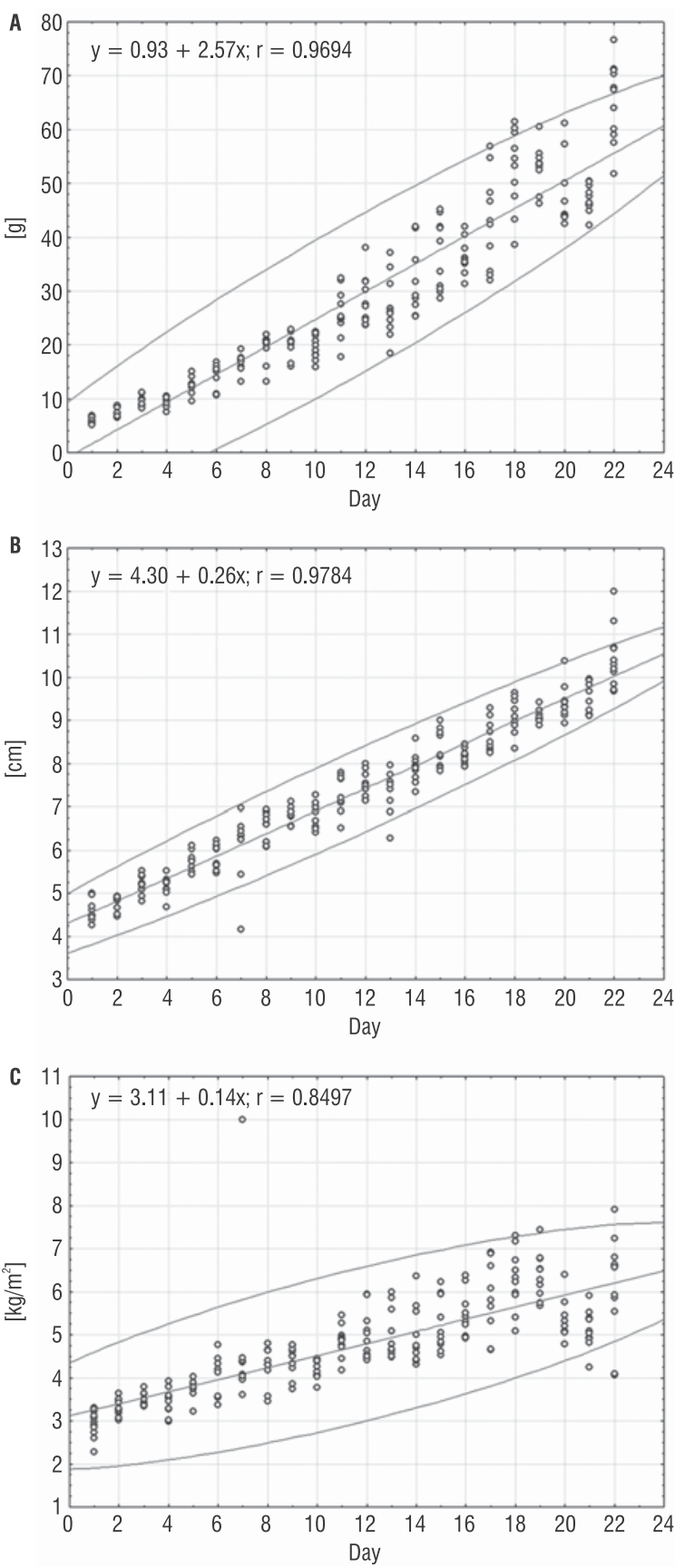

Figure 8. Bony weight (A), length (B) and body mass index (C) of male rat pups during the first 3 weeks of postnatal life. Regression equation and Spearman rank ( $r$ ) are presented on the corresponding graph.

may disturb gestational physiology [11]. Most studies indicated that the highest mineralisation rate, evaluated by alizarin red S staining, is observed in the axial skeleton, particularly in primary ossification centre of the thoracic and lumbar vertebrae and ribs [1, 9-11]. Mineralisation of the other elements - especially cranial bones, that require intramembranous ossification - is characterised by many variations and is
Table 1. Body weight, length and body mass index (BMI) during the first 3 weeks of postnatal life of male rat pup. All data presented as mean \pm standard deviation

\begin{tabular}{llll}
\hline Day & Weight [g] & Length [mm] & BMI [kg/m $]$ \\
\hline 1 & $6.04 \pm 0.57$ & $4.56 \pm 0.24$ & $2.91 \pm 0.30$ \\
2 & $7.62 \pm 0.80$ & $4.79 \pm 0.18$ & $3.31 \pm 0.20$ \\
3 & $9.47 \pm 1.06$ & $5.19 \pm 0.22^{*}$ & $3.51 \pm 0.15$ \\
4 & $9.47 \pm 1.06$ & $5.10 \pm 0.23$ & $3.44 \pm 0.31$ \\
5 & $12.32 \pm 1.67$ & $5.72 \pm 0.24^{*}$ & $3.75 \pm 0.22$ \\
6 & $14.07 \pm 2.41$ & $5.85 \pm 0.28$ & $4.08 \pm 0.45$ \\
7 & $16.79 \pm 1.59$ & $6.17 \pm 0.82$ & $4.69 \pm 1.88^{*}$ \\
8 & $18.19 \pm 3.29$ & $6.51 \pm 0.34$ & $4.26 \pm 0.44$ \\
9 & $20.17 \pm 2.36$ & $6.77 \pm 0.21$ & $4.39 \pm 0.36$ \\
10 & $19.51 \pm 2.27$ & $6.80 \pm 0.28$ & $4.20 \pm 0.22$ \\
11 & $25.92 \pm 4.56^{*}$ & $7.28 \pm 0.41^{*}$ & $4.85 \pm 0.36^{*}$ \\
12 & $28.06 \pm 4.51$ & $7.49 \pm 0.28$ & $4.98 \pm 0.56$ \\
13 & $27.00 \pm 5.75$ & $7.20 \pm 0.48$ & $5.01 \pm 0.58$ \\
14 & $31.24 \pm 6.46^{*}$ & $7.89 \pm 0.34^{*}$ & $4.97 \pm 0.67$ \\
15 & $36.63 \pm 6.58^{*}$ & $8.34 \pm 0.42^{*}$ & $5.23 \pm 0.61$ \\
16 & $36.28 \pm 3.13$ & $8.15 \pm 0.16$ & $5.47 \pm 0.52$ \\
17 & $42.91 \pm 8.82^{*}$ & $8.63 \pm 0.36^{*}$ & $5.73 \pm 0.90$ \\
18 & $51.67 \pm 7.68^{*}$ & $9.07 \pm 0.47^{*}$ & $6.26 \pm 0.67$ \\
19 & $53.06 \pm 4.00$ & $9.12 \pm 0.18$ & $6.38 \pm 0.54$ \\
20 & $47.73 \pm 6.47^{*}$ & $9.42 \pm 0.40$ & $5.36 \pm 0.45^{*}$ \\
21 & $47.18 \pm 2.61$ & $9.62 \pm 0.36$ & $5.12 \pm 0.45$ \\
22 & $65.40 \pm 7.06^{*}$ & $10.44 \pm 0.67^{*}$ & $6.09 \pm 1.15^{*}$ \\
\hline$* 005$ & & &
\end{tabular}

${ }^{*} p<0.05$ vs. previous day

even more sensitive for external factors [11], as well as staining process itself $[6,21,33]$. Alizarin red $\mathrm{S}$ staining may also influence the appendicular skeleton which can present differences between right and left side/paw. Usually, the most visible differences are in distally located elements like phalanges, metacarpal and metatarsal bones [8-11]. Such observations were confirmed for other laboratory animals including mice, rabbits, fishes and birds, as well.

As it was pointed out above, both selected methods are useful in developmental studies. For any new xenobiotic, data obtained with single and/or double staining is obligatory for the human risk and hazard assessment, even staining calibration and validation of bones mineralisation is difficult and results are very subjective. Unlike mineralisation assessment that depends on alizarin red $\mathrm{S}$ absorption, the single method is still a gold standard for evaluation of bony shape $[2,8,11]$. In contrast to staining methods, various 

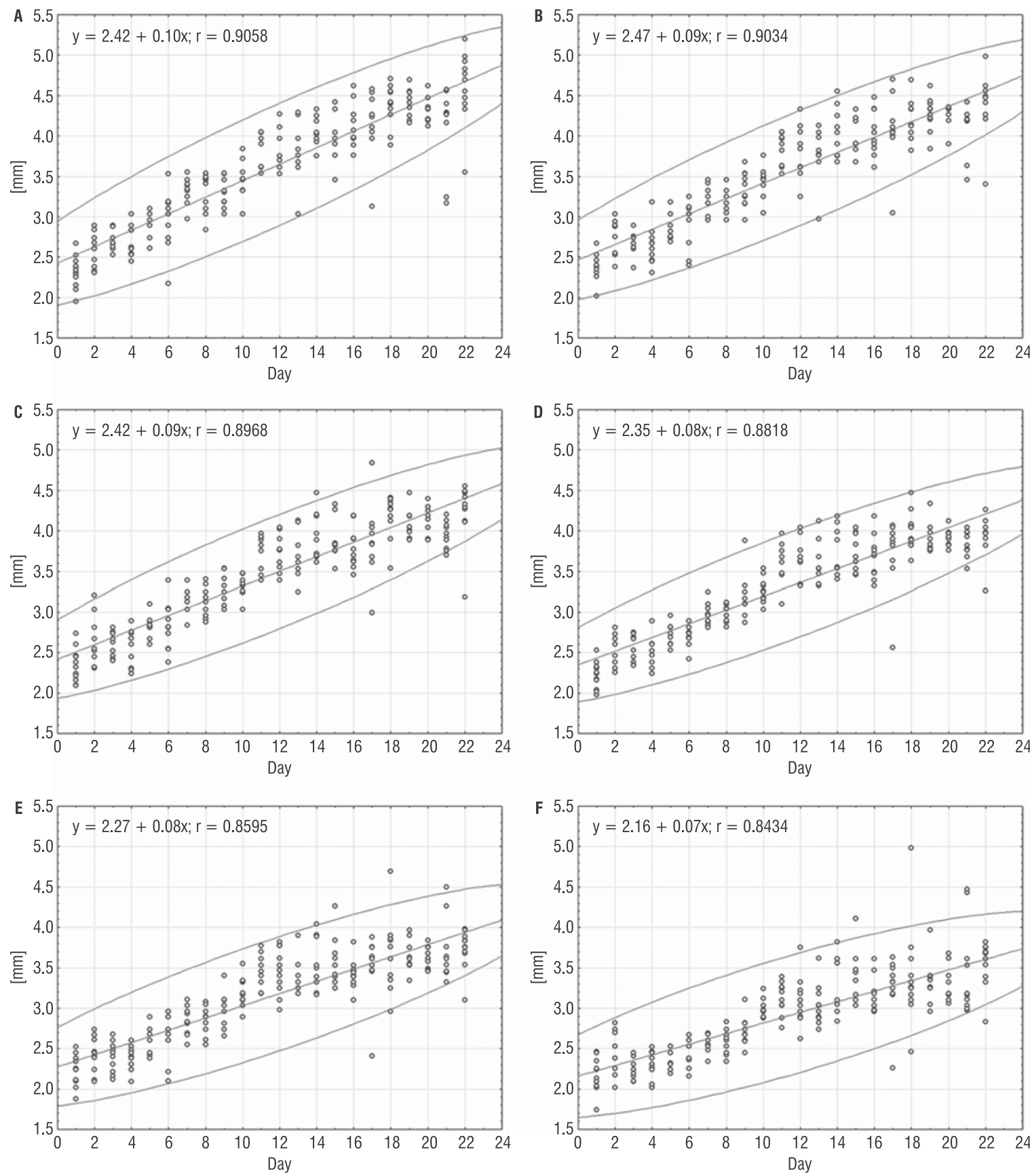

Figure 9. Width of the lumbar vertebrae: L1 (A), L2 (B), L3 (C), L4 (D), L5 (E) and L6 (F); during the first 3 weeks of postnatal life. Regression equation and Spearman rank $(r)$ are presented on the corresponding graph.

radiological examinations are still sporadically used in reproductive studies. The currently applied digital radiography has not any international validation for animal developmental studies but all the preliminary data indicated its usefulness, especially for the quantitative evaluation of the ossification process. Our previous data partially confirmed current results and indicated different degree of vertebral mineralisation. In both untreated animals and exposed in-utero to valproic acid, the optical density of the atlas and axis was higher than lumbar vertebrae and last two thoracic vertebrae $(\mathrm{T} 12, \mathrm{~T} 13)$ in pups examined $12-18 \mathrm{~h}$ post-delivery [6]. However, evaluation of mineralisation of the upper two cervical vertebrae was limited only to primary ossification centres, which are relatively big structures. Similar data was also obtained for rat foetuses and pups, prenatally exposed to various selective (DFU; 5,5-dimethyl-3-(3-fluorophenyl)-4-(4-methylsul- 
Table 2. Width (mm; mean \pm standard deviation) of the lumbar vertebrae (L1-L6) during the first 3 weeks of postnatal life of male rat pup

\begin{tabular}{|c|c|c|c|c|c|c|}
\hline Day & L1 & L2 & L3 & L4 & L5 & L6 \\
\hline 1 & $2.32 \pm 0.19$ & $2.35 \pm 0.19$ & $2.34 \pm 0.20$ & $2.23 \pm 0.16$ & $2.21 \pm 0.19$ & $2.17 \pm 0.21$ \\
\hline 2 & $2.59 \pm 0.21^{*}$ & $2.74 \pm 0.22^{*}$ & $2.67 \pm 0.30^{*}$ & $2.54 \pm 0.18^{*}$ & $2.42 \pm 0.22$ & $2.47 \pm 0.26^{*}$ \\
\hline 3 & $2.70 \pm 0.11$ & $2.70 \pm 0.15$ & $2.61 \pm 0.15$ & $2.56 \pm 0.17$ & $2.39 \pm 0.19$ & $2.26 \pm 0.14$ \\
\hline 4 & $2.66 \pm 0.19$ & $2.64 \pm 0.24$ & $2.57 \pm 0.23$ & $2.47 \pm 0.19$ & $2.40 \pm 0.15$ & $2.30 \pm 0.17$ \\
\hline 5 & $2.91 \pm 0.18^{*}$ & $2.91 \pm 0.17^{*}$ & $2.83 \pm 0.14$ & $2.71 \pm 0.14^{*}$ & $2.57 \pm 0.18$ & $2.37 \pm 0.14$ \\
\hline 6 & $2.95 \pm 0.37$ & $2.92 \pm 0.30$ & $2.82 \pm 0.29$ & $2.72 \pm 0.13$ & $2.61 \pm 0.27$ & $2.45 \pm 0.17$ \\
\hline 7 & $3.31 \pm 0.16^{*}$ & $3.24 \pm 0.16^{*}$ & $3.15 \pm 0.15^{*}$ & $2.97 \pm 0.13^{*}$ & $2.84 \pm 0.17^{*}$ & $2.56 \pm 0.10$ \\
\hline 8 & $3.30 \pm 0.24$ & $3.21 \pm 0.17$ & $3.10 \pm 0.19$ & $2.99 \pm 0.10$ & $2.82 \pm 0.20$ & $2.58 \pm 0.15$ \\
\hline 9 & $3.30 \pm 0.18$ & $3.34 \pm 0.22$ & $3.27 \pm 0.19$ & $3.19 \pm 0.28$ & $2.98 \pm 0.21$ & $2.72 \pm 0.18$ \\
\hline 10 & $3.45 \pm 0.23$ & $3.44 \pm 0.19$ & $3.32 \pm 0.13$ & $3.27 \pm 0.16$ & $3.20 \pm 0.19$ & $3.01 \pm 0.11^{*}$ \\
\hline 11 & $3.85 \pm 0.19^{*}$ & $3.85 \pm 0.19^{*}$ & $3.74 \pm 0.21^{*}$ & $3.61 \pm 0.24^{*}$ & $3.45 \pm 0.20^{*}$ & $3.12 \pm 0.20$ \\
\hline 12 & $3.88 \pm 0.26$ & $3.80 \pm 0.31$ & $3.74 \pm 0.23$ & $3.70 \pm 0.25$ & $3.46 \pm 0.28$ & $3.10 \pm 0.29$ \\
\hline 13 & $3.82 \pm 0.36$ & $3.75 \pm 0.32$ & $3.69 \pm 0.27$ & $3.59 \pm 0.25$ & $3.40 \pm 0.22$ & $3.04 \pm 0.26$ \\
\hline 14 & $4.06 \pm 0.21$ & $4.03 \pm 0.29^{*}$ & $3.91 \pm 0.29 *$ & $3.71 \pm 0.30$ & $3.53 \pm 0.31$ & $3.21 \pm 0.33$ \\
\hline 15 & $4.02 \pm 0.30$ & $3.97 \pm 0.20$ & $3.92 \pm 0.27$ & $3.76 \pm 0.23$ & $3.58 \pm 0.30$ & $3.34 \pm 0.34$ \\
\hline 16 & $4.12 \pm 0.27$ & $4.04 \pm 0.28$ & $3.79 \pm 0.25$ & $3.68 \pm 0.27$ & $3.42 \pm 0.19$ & $3.17 \pm 0.22$ \\
\hline 17 & $4.14 \pm 0.41$ & $4.07 \pm 0.43$ & $3.89 \pm 0.46$ & $3.72 \pm 0.44$ & $3.51 \pm 0.41$ & $3.26 \pm 0.38$ \\
\hline 18 & $4.37 \pm 0.25$ & $4.23 \pm 0.24$ & $4.20 \pm 0.27^{*}$ & $4.01 \pm 0.23^{*}$ & $3.66 \pm 0.45$ & $3.40 \pm 0.62$ \\
\hline 19 & $4.42 \pm 0.17$ & $4.27 \pm 0.21$ & $4.11 \pm 0.17$ & $3.93 \pm 0.18$ & $3.64 \pm 0.19$ & $3.33 \pm 0.27$ \\
\hline 20 & $4.30 \pm 0.15$ & $4.27 \pm 0.14$ & $4.15 \pm 0.17$ & $3.92 \pm 0.10$ & $3.64 \pm 0.14$ & $3.31 \pm 0.21$ \\
\hline 21 & $4.13 \pm 0.51$ & $4.09 \pm 0.30$ & $3.95 \pm 0.17$ & $3.85 \pm 0.16$ & $3.71 \pm 0.38$ & $3.45 \pm 0.57$ \\
\hline 22 & $4.59 \pm 0.42^{*}$ & $4.44 \pm 0.41^{*}$ & $4.27 \pm 0.37^{*}$ & $3.97 \pm 0.28$ & $3.73 \pm 0.23$ & $3.54 \pm 0.28$ \\
\hline
\end{tabular}

${ }^{*} \mathrm{p}<0.05$ vs. previous day

phonyl) phenyl-2(5H)-furanon) and non-selective (ibuprofen, piroxicam, tolmetin) cyclooxygenase inhibitors, but offspring were evaluated on prenatal day 21 and postnatal day $7^{\text {th }}$, respectively $[5,7]$. Even values of the bone density differ from the currently presented ones - probably due to different exposure parameter of X-ray tube - the cranio-caudal mineralisation trend was also revealed. Such data as well as its correlation with body weight was also proved in experimental [12, 20] and epidemiological human studies [27]. However, it should be noted, that in the currently examined animals proportions of pups with unfused vertebral ossification centres on day 22 generally increase from $L 1$ to $L 6$ with the exception of L5. In the light of the fact from previous studies $[12,20]$, such unaccepted finding regarding $5^{\text {th }}$ lumbar vertebrae is probably incidental.

It is worth to point out, that the best advantage of digital radiography is a precision of the mineralisation measurement. In contrary, loss of bone tissue is visible on the classic skeletal X-ray when $30-50 \%$ of bone is resorbed. However, mineralisation could be also analysed using atomic absorption spectroscopy, but the method is rarely applied since it needs expensive equipment and gives only quantitative results [24]. For a long time it was the only method that allowed direct measurement of various elements concentration, but presently such data can be also obtained with mMR [4] or special X-ray microprobe [28]. On the other hand, usefulness of MR is also limited especially in highly mineralised structures, due to low signal in both principal T1- and T2-weighted images [4]. The bone mineralisation could be also evaluated by a single or dual photon absorptiometry which were currently replaced by a single or more commonly used, dual X-ray absorptiometry. From clinical point of view, the last one is a method of choice since it eliminates differences, secondary to presence of various surrounding soft tissues but similar to currently applied system it measure the total density of the region of interest (in case of the vertebral column it will be the density of vertebral body, arch and processes). 
Table 3. Optical density (pixel; mean \pm standard deviation) of the lumbar vertebrae (L1-L6) during the first 3 weeks of postnatal life of male rat pup

\begin{tabular}{|c|c|c|c|c|c|c|}
\hline Day & L1 & L2 & L3 & L4 & L5 & L6 \\
\hline 1 & $121.97 \pm 28.30$ & $119.63 \pm 26.74$ & $120.01 \pm 24.89$ & $117.87 \pm 23.79$ & $119.80 \pm 25.62$ & $121.74 \pm 27.50$ \\
\hline 2 & $146.91 \pm 54.85^{*}$ & $142.84 \pm 51.05^{*}$ & $137.28 \pm 48.65$ & $135.62 \pm 44.42$ & $134.12 \pm 41.98$ & $134.82 \pm 38.61$ \\
\hline 3 & $99.42 \pm 7.43^{*}$ & $99.23 \pm 7.07^{*}$ & $99.34 \pm 6.49^{*}$ & $98.94 \pm 5.49^{*}$ & $97.69 \pm 5.46^{*}$ & $99.38 \pm 4.83^{*}$ \\
\hline 4 & $109.73 \pm 24.56$ & $108.36 \pm 21.60$ & $106.90 \pm 18.25$ & $105.28 \pm 15.40$ & $104.18 \pm 14.65$ & $107.20 \pm 17.79$ \\
\hline 5 & $123.78 \pm 22.85$ & $122.12 \pm 21.60$ & $119.35 \pm 20.40$ & $120.37 \pm 17.91$ & $115.52 \pm 17.69$ & $117.90 \pm 17.93$ \\
\hline 6 & $104.84 \pm 20.73$ & $104.11 \pm 19.25$ & $106.39 \pm 22.04$ & $107.30 \pm 22.43$ & $104.74 \pm 19.98$ & $106.80 \pm 22.17$ \\
\hline 7 & $125.59 \pm 29.65^{*}$ & $123.60 \pm 29.41$ & $123.23 \pm 27.34$ & $121.47 \pm 24.24$ & $120.83 \pm 24.13$ & $124.57 \pm 27.04$ \\
\hline 8 & $107.29 \pm 26.45$ & $107.81 \pm 26.03$ & $107.32 \pm 23.87$ & $107.49 \pm 22.04$ & $106.73 \pm 19.85$ & $108.34 \pm 20.81$ \\
\hline 9 & $114.96 \pm 29.31$ & $114.14 \pm 30.02$ & $112.94 \pm 28.50$ & $113.74 \pm 27.47$ & $112.97 \pm 24.72$ & $114.03 \pm 29.98$ \\
\hline 10 & $103.60 \pm 20.45$ & $103.44 \pm 18.59$ & $103.89 \pm 19.01$ & $104.51 \pm 18.96$ & $105.01 \pm 18.88$ & $105.67 \pm 18.48$ \\
\hline 11 & $114.24 \pm 13.56$ & $114.68 \pm 15.04$ & $115.01 \pm 15.40$ & $116.31 \pm 16.58$ & $117.10 \pm 16.51$ & $118.07 \pm 16.41$ \\
\hline 12 & $109.22 \pm 12.75$ & $107.34 \pm 16.31$ & $107.99 \pm 16.05$ & $109.17 \pm 15.30$ & $109.67 \pm 15.13$ & $110.86 \pm 17.62$ \\
\hline 13 & $108.14 \pm 16.75$ & $110.54 \pm 19.81$ & $114.95 \pm 21.71$ & $116.09 \pm 25.16$ & $114.30 \pm 20.92$ & $115.23 \pm 19.70$ \\
\hline 14 & $116.23 \pm 22.89$ & $117.05 \pm 23.09$ & $118.34 \pm 23.16$ & $118.06 \pm 21.26$ & $118.54 \pm 20.65$ & $120.50 \pm 23.09$ \\
\hline 15 & $101.87 \pm 5.96$ & $102.74 \pm 6.10$ & $103.99 \pm 6.73$ & $105.12 \pm 6.60$ & $106.10 \pm 6.13$ & $107.48 \pm 6.63$ \\
\hline 16 & $113.47 \pm 12.20$ & $114.97 \pm 15.16$ & $116.64 \pm 15.65$ & $116.77 \pm 16.85$ & $117.36 \pm 17.17$ & $119.27 \pm 17.69$ \\
\hline 17 & $116.18 \pm 16.19$ & $118.21 \pm 15.50$ & $119.17 \pm 16.22$ & $121.17 \pm 17.53$ & $123.03 \pm 18.50$ & $127.10 \pm 21.98$ \\
\hline 18 & $126.25 \pm 19.70$ & $127.15 \pm 17.22$ & $126.52 \pm 16.17$ & $126.17 \pm 16.37$ & $127.69 \pm 18.70$ & $130.01 \pm 19.57$ \\
\hline 19 & $130.67 \pm 23.18$ & $134.19 \pm 26.36$ & $136.67 \pm 26.23$ & $139.55 \pm 27.36$ & $142.04 \pm 31.22$ & $144.37 \pm 31.22$ \\
\hline 20 & $123.16 \pm 20.11$ & $123.65 \pm 18.87$ & $122.93 \pm 17.64$ & $124.86 \pm 21.28$ & $124.95 \pm 20.93$ & $127.76 \pm 21.04$ \\
\hline 21 & $115.08 \pm 19.37$ & $114.31 \pm 16.30$ & $113.94 \pm 13.69$ & $115.52 \pm 16.26$ & $116.11 \pm 15.80$ & $117.10 \pm 16.25$ \\
\hline 22 & $143.54 \pm 17.01^{*}$ & $149.46 \pm 18.08 *$ & $151.09 \pm 18.67^{*}$ & $154.45 \pm 18.43^{*}$ & $159.03 \pm 20.48^{*}$ & $163.92 \pm 23.59^{*}$ \\
\hline
\end{tabular}

${ }^{*} p<0.05$ vs. previous day

Some apparatus allow also examination of skeletal morphology and the method is called a morphometric X-ray absorptiometry [13, 28, 29]. However, due to incompatibility with pups of small laboratory animals that is a consequence of low resolution power, it has been used only in late postnatal studies or for pups obtained from large laboratory animals, e.g. lambs or dogs $[13,16,30,31]$. The most promising method is CT, particularly quantitative $\mathrm{mCT}$. Unlike dual X-ray absorptiometry and currently discussed digital radiography, it allows determination of a direct volumetric mineral density, not a summary surface one. Mico CT is also the only method that allows concomitant 3-dimensional skeletal visualisation and bone microarchitecture examination $[5,6,18,30]$.

The main limitation of this study is the relatively low number of evaluated animals on each postnatal day ( $n=9-12)$. However, normal distribution of the obtained results may indicate data repro- ducibility. On the other hand, the mineralisation was evaluated only in male pups, just to omit any hormonal and developmental sex differences, and may be slightly different in females. Moreover, all the animals were without any developmental abnormalities, including signs of intrauterine growth retardation; and for this reason a mean density for the whole rat population may be different as well. Unlike in typical developmental and reproductive toxicological studies, maternal effect could be withdrawn or minimal since all pups were taken from untreated control litters and all dams were kept under physiological condition. On the other hand, the applied methodology seems to be useful for developmental studies, however due to radiation it cannot be routinely applied for day by day longitudinal evaluation. For this reason, the follow up of individual pups was not performed. However, since the method described is relatively cheap and 

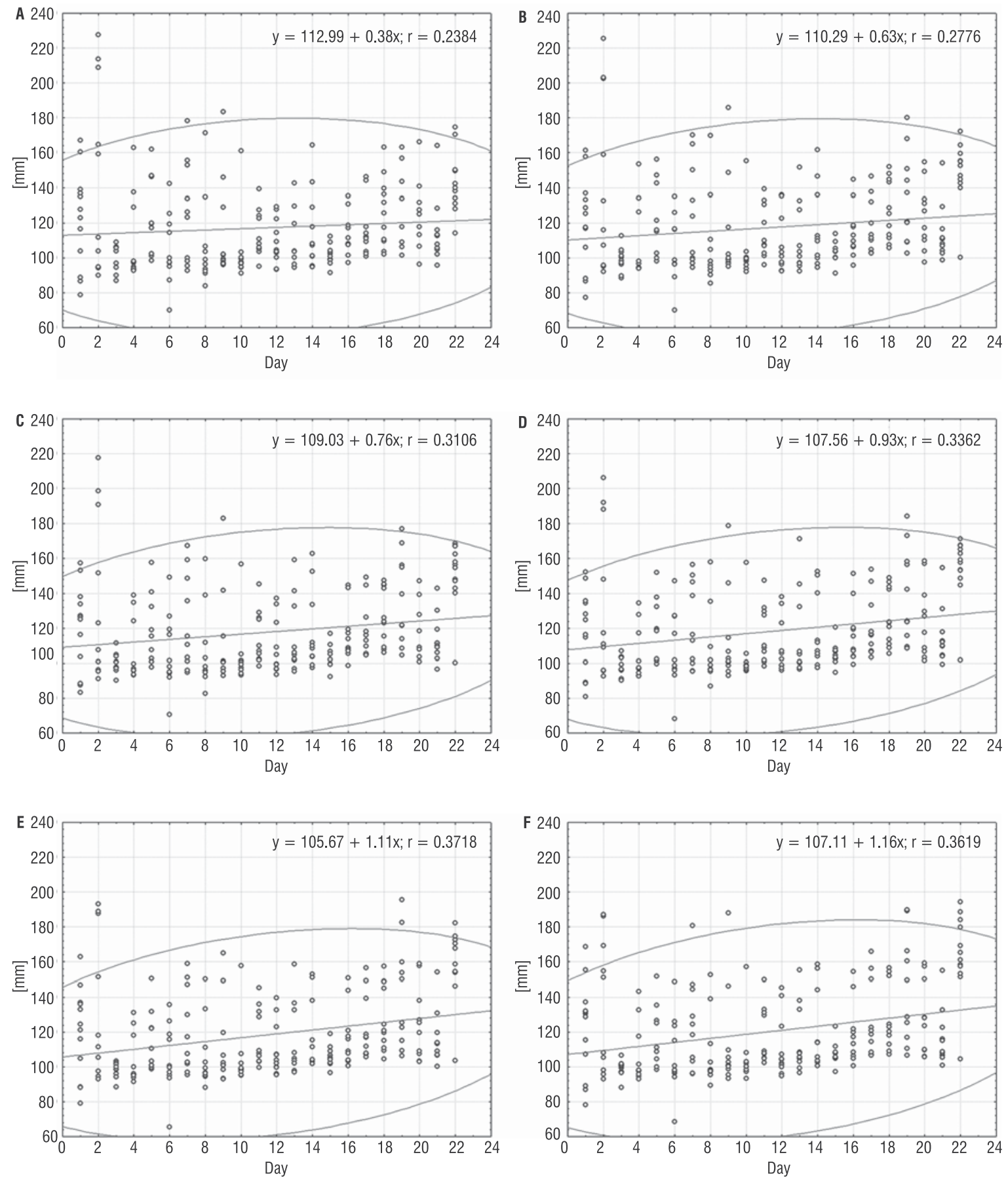

Figure 10. Optical density of the lumbar vertebrae: L1 (A), L2 (B), L3 (C), L4 (D), L5 (E) and L6 (F); during the first 3 weeks of postnatal life. Regression equation and Spearman rank ( $r$ ) are presented on the corresponding graph.

easy, it could be supplementary to alizarin red $\mathrm{S}$ staining as it provides both qualitative and quantitative data on mineralisation. The obtained results may strongly improve a classic embryo-foetal data, especially for multigenerational studies.

\section{CONCLUSIONS}

Development of rat lumbar vertebrae is a dynamic process in an early postnatal period. Unlike vertebral mineralisation, pups' body weight and length as well as vertebral width increased during the postnatal 


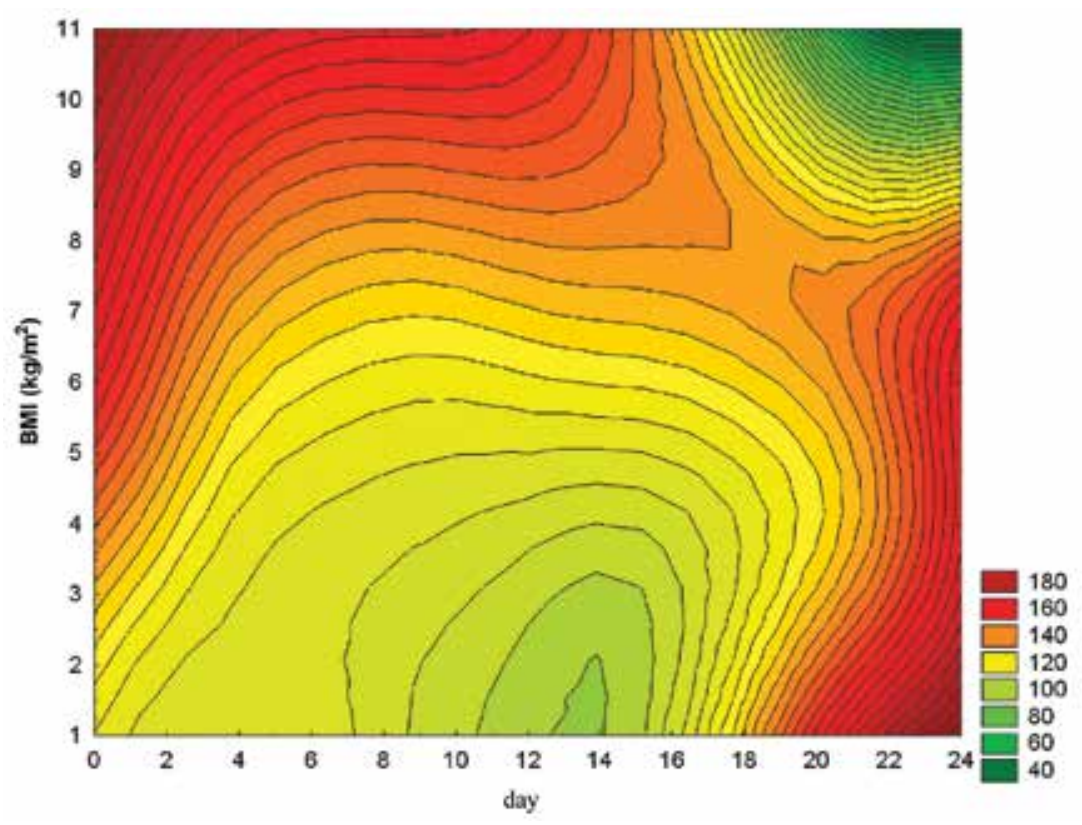

Figure 11. Total optical density (pixel on the colour scale) of lumbar part of the vertebral column in relation to pup's body mass index (BMI) and age, examined simultaneously.

period and significantly correlated with their age. The complete fusion of the primary ossification centres had a cranio-caudal direction and started on day 19 after parturition and for the upper 3 lumbar vertebrae was completed by day 22 .

\section{REFERENCES}

1. Aliverti V, Bonanomi L, Giavini E, Leone VG, Mariani L (1979) The extent of fetal ossification as an index of delayed development in teratogenic studies on the rat. Teratology, 20: 237-242.

2. Barrow PC (2009) Reproductive toxicity testing for pharmaceuticals under ICH. Reprod Toxicol, 28: 172-179.

3. Boardman JP, Mitala JJ, Carrano RA, Iuliucci JD (1984) Cartilage-staining technique for the examination of unskinned fetal rat specimens previously processed with alizarin red $\mathrm{S}$. Teratology, 30: 383-384.

4. Bulas D, Egloff A (2013) Benefits and risks of MRI in pregnancy. Semin Perinatol, 37: 301-304.

5. Burdan F, Pliszczynska-Steuden M, Rozylo-Kalinowska I, Chalas A, Rozylo TK, Staroslawska E, Klepacz R, Szumilo J (2011) Developmental outcome after exposure to cyclooxygenase inhibitors during pregnancy and lactation. Reprod Toxicol, 32: 407-417.

6. Burdan F, Rozylo-Kalinowska I, Rozylo TK, Chahoud I (2002) A new rapid radiological procedure for routine teratological use in bone ossification assessment: a supplement for staining methods. Teratology, 66: 315-325.

7. Burdan F, Rozylo-Kalinowska I, Szumilo J, Dudka J, Klepacz R (2008) Cyclooxygenase inhibitors affect bone mineralization in rat fetuses. Cells Tissues Organs, 187: 221-232.

8. Burdan F, Szumilo J, Dudka J, Klepacz R, Blaszczak M, Solecki M, Korobowicz A, Chalas A, Klepacki J, Palczak M, Zuchnik-Wrona A, Hadała-Kis A, Urbanowicz Z, Wojtowicz Z
(2005) Morphological studies in modern teratological investigations. Folia Morphol, 64: 1-8.

9. Chahoud I, Paumgartten FJ (2009) Dose-response relationships of rat fetal skeleton variations: Relevance for risk assessment. Environ Res, 109: 922-929.

10. Chahoud I, Paumgartten FJ (2009) Influence of litter size on the postnatal growth of rat pups: is there a rationale for litter-size standardization in toxicity studies? Environ Res, 109: 1021-1027.

11. Christian MS (2001) Test methods for assessing female reproductive and developmental toxicology. In: Hayes AW ed. Principles and method of toxicology. 4 Ed. Taylor and Francis, Philadelphia, pp. 1301-1381.

12. Czyz M, Kedzia A (2004) Geometrical assessment of the foetal lumbar vertebral column - clinical implications. Folia Morphol, 63: 431-438.

13. Damilakis J, Perisinakis K, Vrahoriti H, Kontakis G, Varveris $\mathrm{H}$, Gourtsoyiannis N (2002) Embryo/fetus radiation dose and risk from dual $\mathrm{X}$-ray absorptiometry examinations. Osteoporos Int, 13: 716-722.

14. De Schaepdrijver L, Delille P, Geys H, Boehringer-Shahidi C, Vanhove C (2014) In vivo longitudinal micro-CT study of bent long limb bones in rat offspring. Reprod Toxicol, 46: 91-97.

15. Environmental Protection (1996). Guidelines for reproductive toxicity risk assessment. Fed Regist, 61: 56274-56322. http://www.epa.gov/raf/publications/pdfs/REPRO51.PDF. Accessed 22 June 2014.

16. Firth EC, Rogers CW, Vickers $M$, Kenyon PR, Jenkinson CM, Blair HT, Johnson PL, Mackenzie DD, Peterson SW, Morris ST (2008) The bone-muscle ratio of fetal lambs is affected more by maternal nutrition during pregnancy than by maternal size. Am J Physiol Regul Integr Comp Physiol, 294: R1890-R18904.

17. French JM (2014) Imaging and morphology in reproductive toxicology: progress to date and future directions. Reprod Toxicol, 48: 37-40. 
18. Guldberg RE, Lin AS, Coleman R, Robertson G, Duvall C. (2004) Microcomputed tomography imaging of skeletal development and growth. Birth Defects Res C Embryo Today, 72: 250-259.

19. International Conference on Harmonisation of Technical Requirements for Registration of Pharmaceuticals for Human Use (1993) Detection of toxicity to reproduction for medical products and toxicity to male fertility. Parent Guideline dated 24 June 1993 (addendum dated 9 November 2000 incorporated in November 2005) http://www.ich. org/products/guidelines/safety/article/safety-guidelines. html. Accessed 22 June, 2014.

20. Kedzia A, Czyz M (2003) Ossification process and lumbar spine morphology in the prenatal period. Med Sci Monit, 9: BR343-BR350.

21. Kimmel CA, Trammell C (1981) A rapid procedure for routine double staining of cartilage and bone in fetal and adult animals. Stain Technol, 56: 271-273.

22. Martin RJ, Fanaroff AA, Walsh MC (2010) Fanaroff and Martin's neonatal-perinatal medicine: diseases of the fetus and infant. $9^{\text {th }}$ Ed. Elsevier, St. Louis:.

23. Menegola E, Broccia ML, Di Renzo F, Giavini E (2002) Comparative study of sodium valproate-induced skeletal malformations using single or double staining methods. Reprod Toxicol, 16: 815-823.

24. Nakamoto T, Grant S, Yazdani M (1989) The effects of maternal caffeine intake during pregnancy on mineral contents of fetal rat bone. Res Exp Med (Berl), 189: 275-280.

25. Oest ME, Jones JC, Hatfield C, Prater MR (2008) Micro-CT evaluation of murine fetal skeletal development yields greater morphometric precision over traditional clear-staining methods. Birth Defects Res B Dev Reprod Toxicol, 83: 582-589.

26. Organization for Economic Co-operation and Development (1983). OECD guideline for testing of chemicals. www.oecd.org/dataoecd/18/12/1948458.pdf. Accessed 22, 2014.

27. Reid IR (2002) Relationships among body mass, its components, and bone. Bone, 31: 547-555.

28. Tomaszewski M, Olchowik G, Tomaszewska M, Burdan F (2012) Use of X-ray microprobe to diagnose bone tissue demineralization after caffeine administration. Folia Histochem Cytobiol, 50: 436-443.

29. Ward KA, Cotton J, Adams JE (2003) A technical and clinical evaluation of digital $\mathrm{X}$-ray radiogrammetry. Osteoporos Int, 14: 389-395.

30. Wise LD, Winkelmann CT (2009) Micro-computed tomography and alizarin red evaluations of boric acid-induced fetal skeletal changes in Sprague-Dawley rats. Birth Defects Res B Dev Reprod Toxicol, 86: 214-219.

31. Wise LD, Xue D, Winkelmann CT (2010) Micro-computed tomographic evaluation of fetal skeletal changes induced by all-trans-retinoic acid in rats and rabbits. Birth Defects Res B Dev Reprod Toxicol, 89: 408-417.

32. Yamada T (1991) Selective staining methods for cartilage of rat fetal specimens previously treated with alizarin red $\mathrm{S}$. Teratology, 43: 615-619.

33. Young AD, Phipps DE, Astroff AB (2000) Large-scale double-staining of rat fetal skeletons using alizarin Red $S$ and alcian blue. Teratology, 61: 273-276. 